\title{
Editorial
}

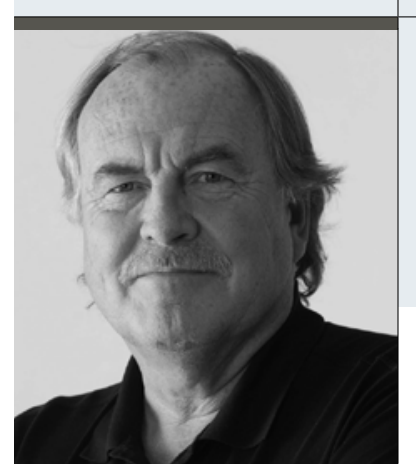

„ICh bin sehr erfreut über diese Entscheidung der WHO, die erst nach langem und zähem Ringen zustande gekommen ist."

Prof. Dr. med. Dr. h.c. Werner Hacke

Seniorprofessor der medizinischen Fakultät der Ruprecht-Karls-Universität Heidelberg, Präsident der World Stroke Organization (WSO)

\section{Der Schlaganfall ist und bleibt eine neurologische Erkrankung!}

$\mathrm{D}$ er WHO-Diagnoseschlüssel für die neue "International Classification of Diseases" (ICD-11) gruppiert den Schlaganfall endgültig zu den neurologischen Erkrankungen. Diese lang überfällige Klassifizierung im ICD-11 ist mehr als angemessen und aus der Sicht der DGN eindeutig zu begrüßen. An der 11. Revision des WHO-Diagnoseschlüssels ICD wird seit 2009 gearbeitet. Sie soll den medizinisch-wissenschaftlichen Fortschritt berücksichtigen und wird 2018 in Kraft treten.

Die „Topic Advisory Group Neurology“ (TAGN), mit ihrem Leiter Professor Raad Shakir, ist während des gesamten Diskussionsprozesses über den ICD-11 dafür eingetreten, dass alle Formen von Schlaganfall in einem Abschnitt zusammengefasst werden und dem Kapitel über neurologische Erkrankungen zugeordnet werden sollen. Alle Symptome zerebrovaskulärer Erkrankungen stehen in einem Zusammenhang mit Funktionsstörungen des Gehirns. Die TAGN hat deshalb bereits im April 2011 der WHO in Genf vorgeschlagen, den Abschnitt „Zerebrovaskuläre Erkrankungen“ mit allen Formen des Schlaganfalls von den HerzKreislauf-Erkrankungen zu den neurologischen Erkrankungen und damit von der Inneren Medizin in die Neurologie zu verschieben. So war es bis zum Herbst 2016 in der Beta-Version des ICD-11 auch vorgesehen. Im letzten September jedoch machte die WHO-Klassifikationsgruppe eine abrupte Kehrtwende: Für uns Experten völlig überraschend wurde der neu geschaffene Abschnitt „Zerebrovaskuläre Erkrankungen" von den neurologischen wieder zu den Herz-Kreislauf-Erkrankungen verschoben.

Die WHO-Entscheidung wurde von intensiven Diskussionen in der internationalen neurologischen Gemeinschaft begleitet. Im Oktober 2016 zeigten sich führende internationale Neurologen und Schlaganfallexperten unter der Federführung von Raad Shakir und Bo Norrving in einem Editorial in Lancet besorgt über die Änderungen im Entwurf für den neuen internationalen Diagnosenschlüssel ICD-11 [1]. Nur dem großen Einsatz der Vertreter der Weltföderation für Neurologie (WFN), Professor Raad Shakir, und der World Stroke Organization (WSO), Professor Bo Norrving, ist es zu verdanken, dass gegen den erheblichen Widerstand einzelner Fachgesellschaften nun doch eine endgültige Entscheidung zugunsten der Neurologie getroffen wurde und die zerebrovaskulären Erkrankungen unter den neurologischen Erkrankungen eingeordnet werden.

Wir alle freuen uns, dass diese Zusammenhänge entsprechend anerkannt wurden und dazu führten, dass das WHO-Team entsprechende Änderungen in der Letztfassung des ICD-11 implementiert hat. Und wir hoffen, dass dieser Paradigmenwechsel große Auswirkungen auf die Medizinpolitik sowie die Forschungsförderung haben wird. Diese Entscheidung zur angemessenen Klassifikation des Schlaganfalls ist auch im Interesse der betroffenen Patienten, denn nun beruhen Ressourcenplanung und Finanzierung auf korrekten Daten, was bisher nicht der Fall war.

Mit kollegialen Grüßen

Werner Hacke

1. Shakir R, Davis $S$, Norrving B, et al. Revising the ICD: stroke is a brain disease. Lancet 2016; 388 (10059): 2475-6 